\title{
Design and Realization of Intelligent Upgrade Based on Temperature Control of Electric Heating Cooker
}

\author{
Li CHEN \\ Department of Instrument Science and Engineering \\ Shanghai Jiao Tong University \\ Shanghai, China \\ E-mail: chenli_damon@sjtu.edu.cn
}

\section{Hao MI}

Department of Instrument Science and Engineering

Shanghai Jiao Tong University

Shanghai, China

E-mail: 553994944@qq.com

\author{
Junzhi CHEN \\ Anqing Marine Appliances Limited Liability Company \\ CSSC \\ Anqing, China \\ E-mail: 485jyb@163.com
}

\author{
Ming YANG* \\ Department of Instrument Science and Engineering \\ Shanghai Jiao Tong University \\ Shanghai, China \\ E-mail: myang@sjtu.edu.cn \\ $+*$ Corresponding author
}

\begin{abstract}
This paper presents the design and realization of intelligent upgrade based on temperature control of electric heating cooker. The cooker is supplied by segmented PID algorithm which helps to realize precise temperature control. By fully utilizing STM32 MCU, intelligent upgrade including smart-friendly interaction as well as pre-stored menu information is achieved, which means automatic temperature control and operation reminding are available. More importantly, the cooker allows manual adjustment during automatic control and can record this information via Flash. In that way, it can implement real-time menu information update, thus making it more human-friendly.
\end{abstract}

Keywords-intelligent upgrade; electric heating cooker; temperature control; menu information pre-store; real-time information update

\section{INTRODUCTION}

Essentially speaking, the traditional electric heating cooker is a temperature control system based on resistance heating which is burned with large inertia and delay [1]. While in actual using process, many repeated operation will happen due to lack of control intelligence. For example, when a cook is making a specific dish, the relationship between temperature and time of cooker is nearly the same, which means the cook needs to do the same operation to control the cooker's temperature output whenever he makes that dish [2]. To some extent, this is a waste of human resource.

To solve this problem, a new intelligent electric heating cooker is designed and realized. For one thing, this cooker can implement precise temperature control just like traditional cooker [3]. For another, it can fully utilize the powerful function of STM32 MCU to achieve intelligent interaction. Meanwhile, by setting pre-stored menu information, in actual using process, the cooker can automatically control the temperature and do some operation reminding. What's more, because of the power down protection of Flash [4], the real-time adjustment is permitted during the automatic control and this adjustment information will be recorded so that the automatic control process will be updated in next operation period based on the adjustment.

In section II, principle of the system is introduced, which includes hardware composition, control algorithm of electric heating cooker and principle of intelligent upgrade. Then in section III, we will mainly discuss the intelligent upgrade of the electric heating cooker. Some intelligent interactions are illustrated and some intelligent functions are introduced. More importantly, the real-time menu information update will be demonstrated. Next, experiment result is analyzed in section $\mathrm{V}$ and a conclusion is drawn in section VI.

\section{PRINCIPLE OF SYSTEM}

\section{A. Hareware Composition}

The hardware part of temperature control system is mainly composed of following module: master control, temperature detect, temperature control, human-computer interaction and controlled objective [1].

The connection relationship among these modules is shown in Fig. 1.

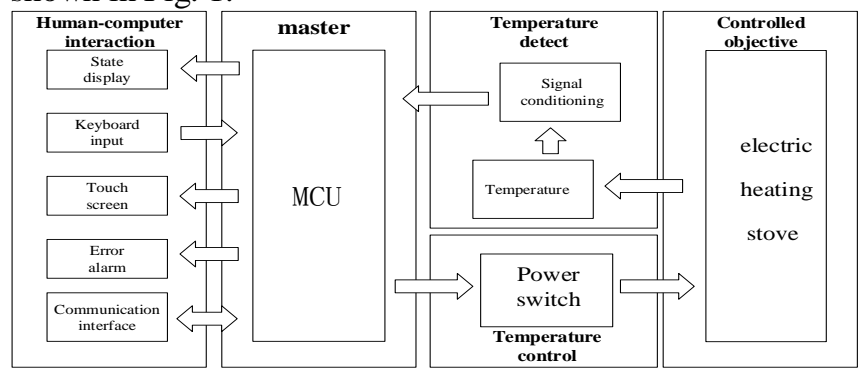

Figure 1. Connection relationship of hardware module 
Master control part is STM32F103 series MCU. Temperature detect module includes type k-thermocouple and MAX6675 chip. Another key part is temperature control module. We choose SSR (solid state relay) as the core part of this module [5]. In our design, we realize power modulation by adjusting PWM pulse width [6]. The principle is shown in Fig. 2.

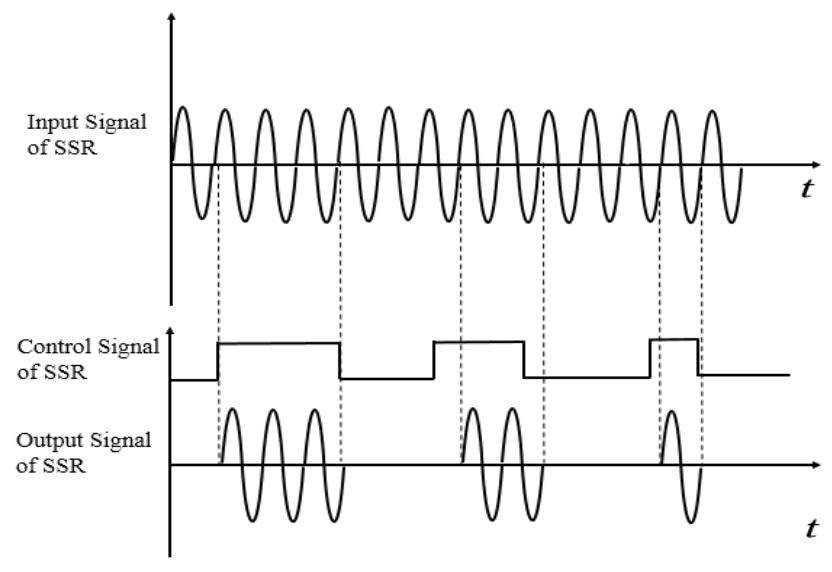

Figure 2. Three singal of SSR

In human-computer interaction module, we use 2.8 inch touch screen, whose location of touch point is determined by the output voltage in abscissa and ordinate.

\section{B. Segmented PID Control Algorithm}

Regular PID control algorithm is one item of the classic control algorithm [7]. While in order to eliminate the potential overshoot and oscillation caused by integral item accumulation, a segmented PID control algorithm is proposed in [8]. Specifically speaking, when the error value is relatively large, we use PD control. When the error value reduces to a specific scope, incremental type PID is used.

Meanwhile, because of the big inertia and delay of the heating system, SSR is turned on fully when the error value is extremely large and turned off if overshoot appears [9]. Considering all of these, the segmented PID control algorithm of this paper is formed whose principle is shown as Fig. 3.

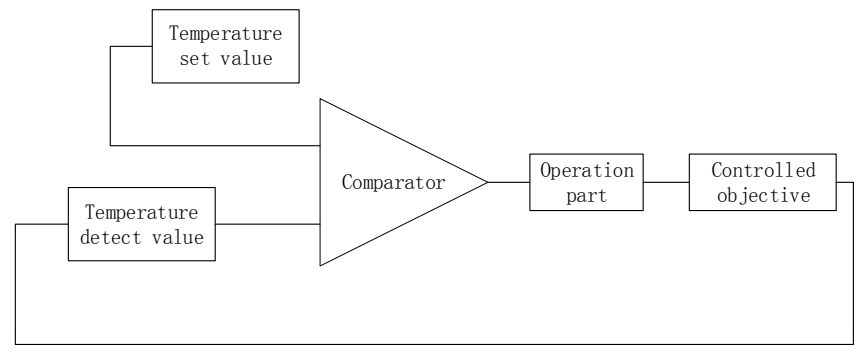

Figure 3. Principle of segmented PID

In Fig. 3 we add a comparator that can decide which control algorithm should be used at present stage according to the error value. We achieve critical parameters as follow by experiment:
1) When the error value is larger than $30 \%$ of the set value, SSR is fully turned on.

2) When the error value is between 10\% and 30\% of the set value, PD control algorithm is applied.

3) When the error value is between zero and $10 \%$ of the set value, incremental type PID control algorithm is applied.

4) When error value is negative, SSR is turned off.

\section{Principle of Intelligent Upgrade}

According to Professor Nelson in Stanford University Artificial Intelligent Research Center, Artificial Intelligent is that machine has some function that is related to people's intelligent, just like judgement, reasoning, identify learning and problem solve [10]. As for our intelligent upgrade, we give our cooker the ability of identify learning. In every new operation period, the cooker can identify whether there is adjustment in last period. And then, it can update the adjustment information, namely, the process learning.

\section{INTELLIGENT UPGRADE OF ELECTRIC HEATING COOKER}

\section{A. Temperature-time Relationship Pre-store}

As we have discussed before, there are many repeated temperature-time alteration process when traditional electric heating cooker are used.

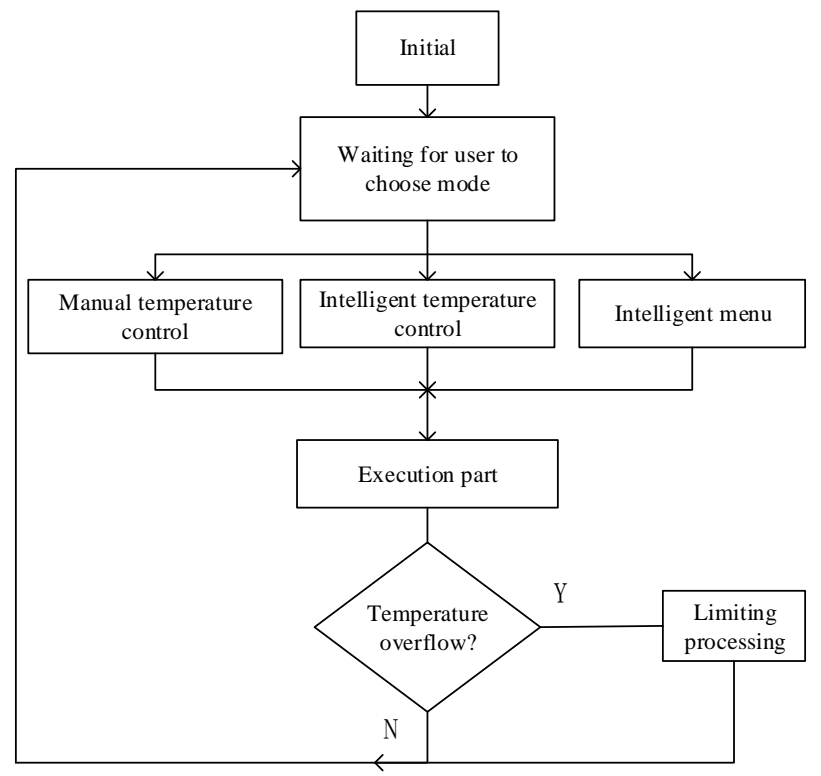

Figure 4. Flow chart of intelligent update

Therefore the purpose of our intelligent upgrade about electric heating cooker is to pre-store these temperature-time alteration relationship information into controller, as well as corresponding operation information. In that way, during the whole cooking process the temperature of the electric heating cooker will change correspondingly at particular time point. At the same time, the corresponding operation information 
will also appear. The specific flow chart is illustrated in Fig. 4.

In manual temperature control mode, the temperature of the electric is decided by manual operation as shown in Fig. 8 , which is just the same as traditional electric heating cooker. Manual temperature control is realized by changing duty cycle of PWM wave generated by MCU.

In intelligent temperature control mode, users can directly select corresponding heating mode according to actual requirement. After selection, the temperature is set automatically. As mentioned in [11], the most common cooking mode is stir fry, stew and temperature preserving. The manual control page and selection page is shown as Fig. 5 .

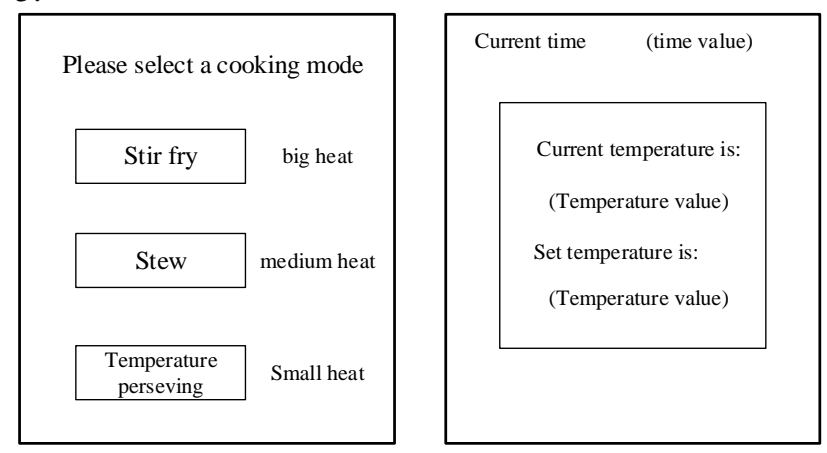

Figure 5. Manual control page and selection page of cooking mode

In intelligent menu mode, users can select one menu according to their demand. After selection, the cooker can automatically running with a particular temperature-time relationship.

Meanwhile, there are some operation tips at different time point. All of these information are pre-stored into $\mathrm{MCU}$, what the users need to do is just to do operation following those tips. Menu selection page and temperature control page is shown as Fig. 6.

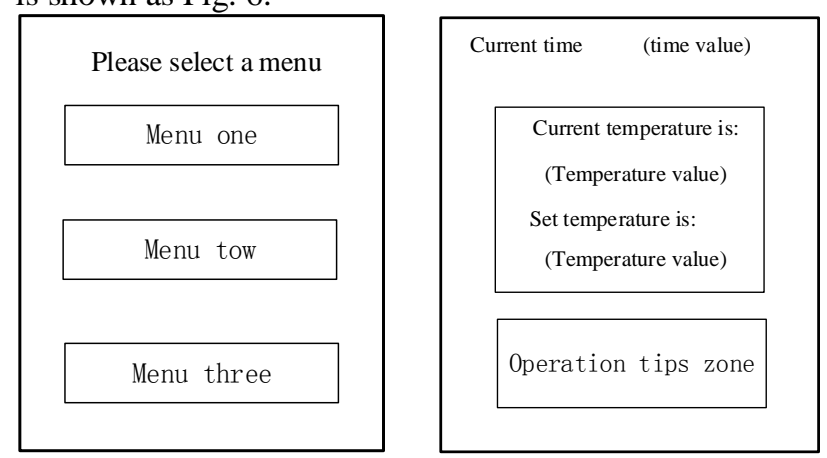

Figure 6. Menu selection and temperature control page

\section{B. Real-time Adjustment Information Update}

Actually we know, no matter in intelligent temperature control mode or in intelligent menu mode, the pre-stored menu information does not always meet to the cook's requirement, therefore manual adjustment is allowed in both intelligent mode. What's more, with the principle that the manual adjustment has higher priority than the pre-stored menu information, update of pre-stored information should be made as long as manual adjustment happens. In that way, we need store manual adjustment information.

Considering our cooker requires data power down protection, we choose Flash as our storage media for which the data still exist when power down happens and erase operation can repeat for many times as well as read and write operation [4]. For our master control module STM32F103RCT6, the capacity of its Flash is 256K, which is enough for our information update.

The principle of real-time manual adjustment update is illustrated as Fig. 7.

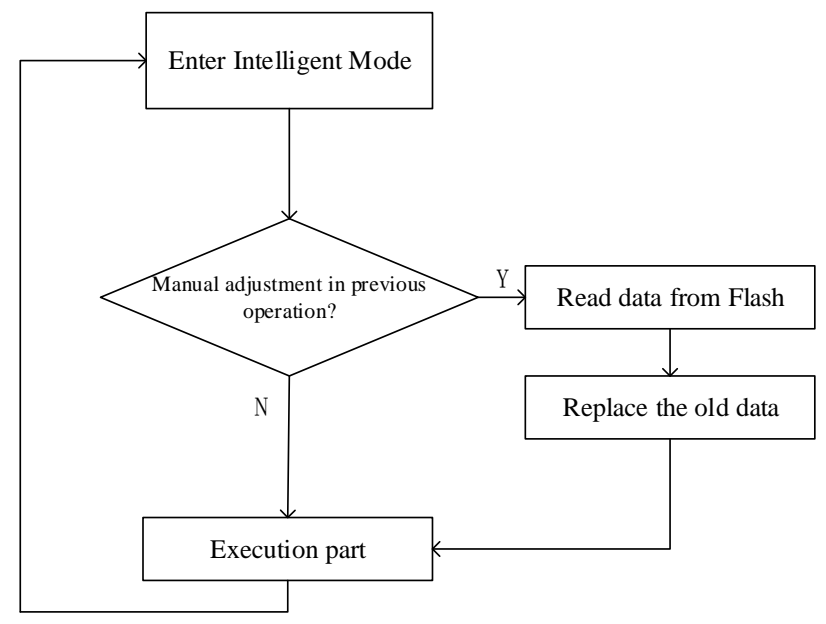

Figure 7. Flow chart of real-time manual adjustment update

As shown in Fig. 7, if the adjustment exists, some data is read from Flash, and the old data is replaced. Otherwise, the execution part works normally. When a manual adjustment happens, the system enters into interrupt mode, in which the temperature set value is changed and final set value is written into Flash. In that way, the menu information in intelligent mode is updated.

\section{EXPERIMENT RESULT AND ANALYSIS}

To verify the effect of segmented PID algorithm, we connect the hardware part as Fig. 1

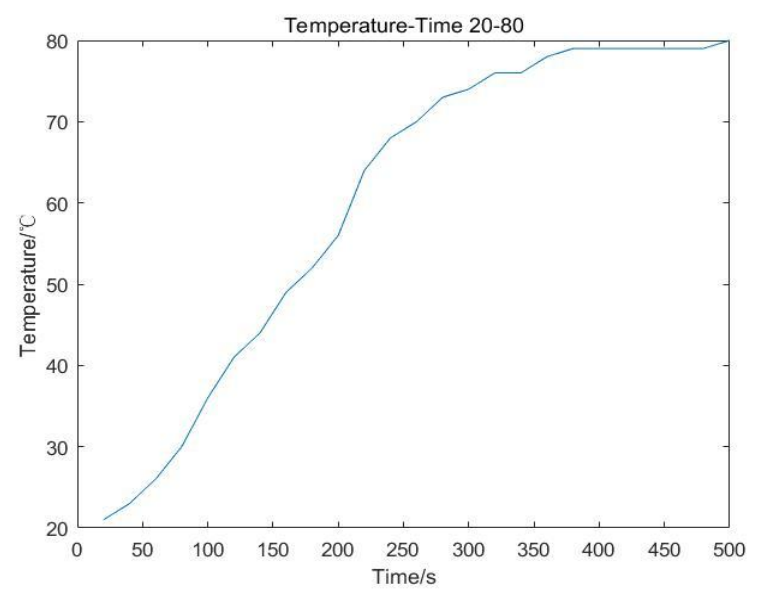

Figure 8. Temperature rise curve of experiment 1 
We control the temperature of the cooker to $80^{\circ} \mathrm{C}$ from ambient temperature. The experiment result is shown in Fig. 8.

Then in order to verify the effect of set value variation, we change the set value from $80^{\circ} \mathrm{C}$ to $100^{\circ} \mathrm{C}$, and the experiment result is demonstrated as Fig. 9.

From Fig. 8 we can see that in the beginning stage the temperature rise very fast, and at the point about $56^{\circ} \mathrm{C}$ the curve rise kind of slowly. When the current temperature is very closely to set value, the curve slows down significantly. At the end of the stage, there is no overshoot which is especially important to a system with large inertia and delay.

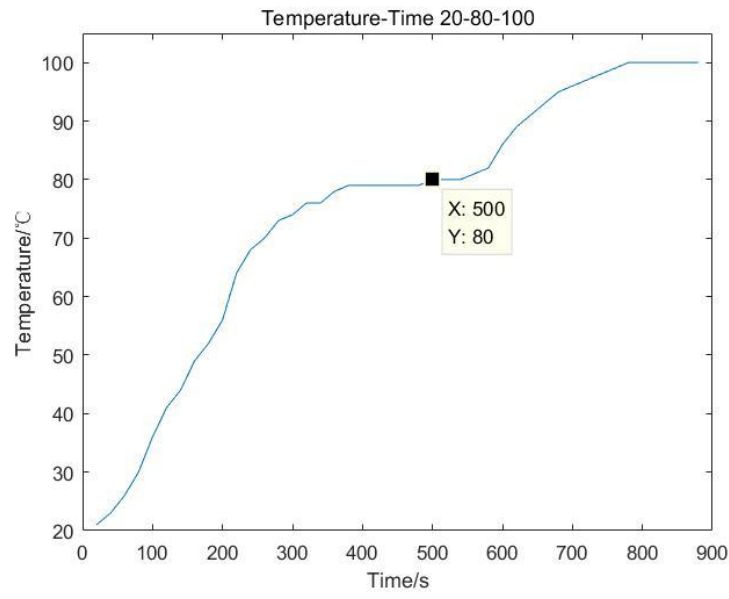

Figure 9. Temperature rise curve when set value varies to $100^{\circ} \mathrm{C}$

Fig. 9 tells us that when the set value is changed there is also no overshoot happen, which is better than the result of regular position type PID control algorithm.

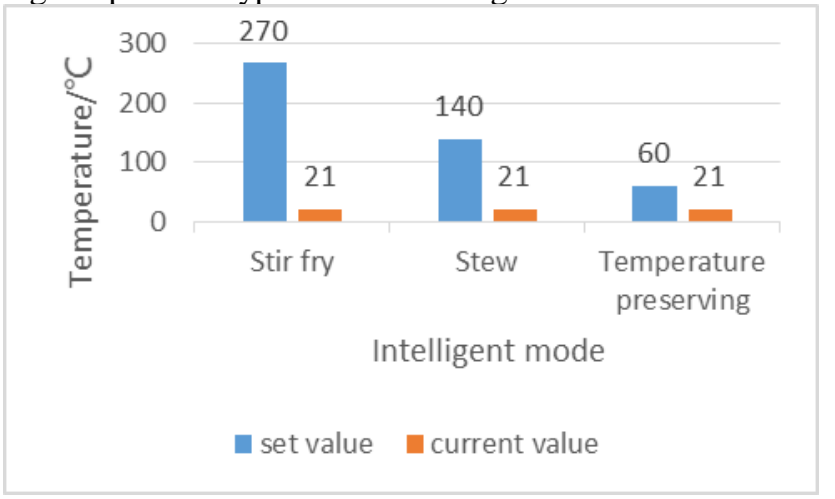

Figure 10. Temperature value of every cooking mode

When we select intelligent temperature control mode, the value of current temperature and set temperature in every cooking mode are both recorded and illustrated as Fig. 10 above.

To verify the function of intelligent menu mode, the variation relationship between set temperature value and time are both recorded and shown in Fig. 11.

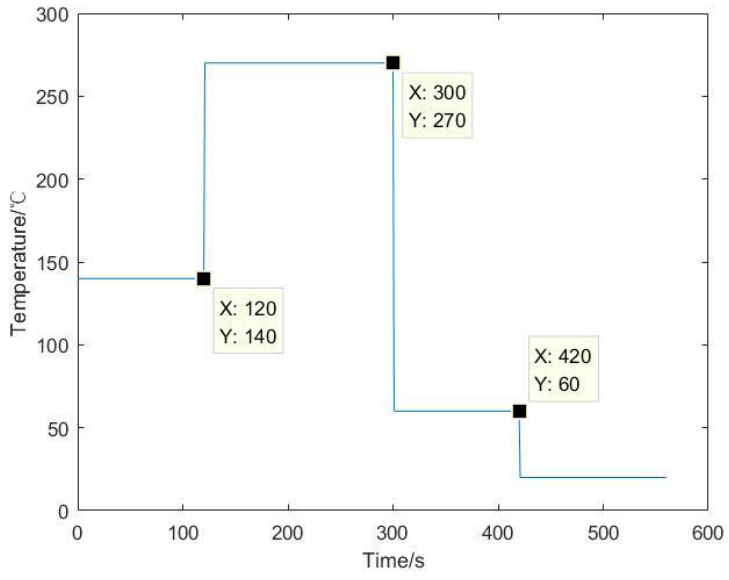

Figure 11. Variation relationship between set temperature value and time

Then we change set value of the first stage and the third stage when the system is running. At the next operation period, the relationship between set temperature value and time are also recorded and shown in Fig. 12.

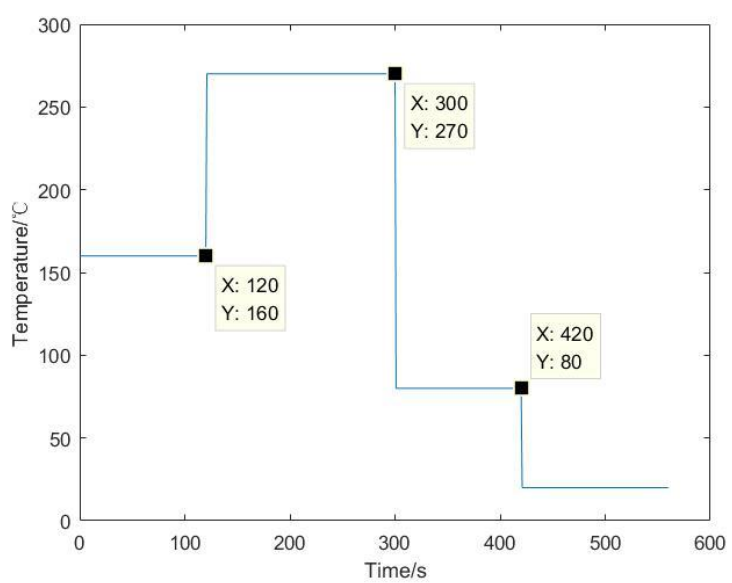

Figure 12. Variation relationship between set temperature value and time

Fig. 11 and Fig. 12 show that the function of real-time manual adjustment update is feasible.

\section{CONCLUSION AND FutURE WORK}

In this paper, we design and implement intelligent upgrade based on temperature control of electric heating cooker. Compared with traditional electric heating cooker, the proposed method can not only realize precise temperature control but also realize intelligent cooking. After intelligent upgrade, our cooker can set temperature value automatically and the relationship between temperature and time can also be set automatically according to different menu which is pre-stored. More importantly, manual adjustment is allowed in intelligent mode and relevant information will be recorded so that temperature-time relationship can be updated at next operation period correspondingly.

While in so many situations, the temperature-time relationship is hard to drawn from menu information. Thus 
cooker's self-learning is necessary. In our future work, we aim to record the relationship between current temperature and time, and then analyze the process to achieve the regulation between set temperature and time. In that way, the intelligent electric heating cooking can do self-learning to achieve menu information, which is a more advanced optimization of human resource.

\section{REFERENCES}

[1] F. B. Yu and W. T. Li, "Design of the Resistance Furnace Intelligent Temperature Controller,"Inner Mongolia University of Science and Technology. Baotou , pp. 8-25, May 30th 2012.

[2] L. Deng, "Method to convert manual cuisine into automatic operations based on time-temperature integrators", Transactions of the CSAE, vol. 29, No 6, March 2013, pp.287-292.

[3] X. L. Chen, "Design and Research on New Intelligent Temperature Control System for stove," Journal of China University of Mining. Beijing, vol. 11, No. 2, December 2001, pp 217-220.

[4] J. L. Zhou, R. F. Luo and C. C. Sun, "Design of data collection and storage system based on STM32," Information Technology, vol. 8, 2016, doi:10.13247/j.cnki.hdzj.2016.08.034.

[5] Y. J. Wang and J. J. Zhu, "SSR and Its Application in SCM Measuring and Controlling System," Journal of South-Central
University for Nationalities. Wuhan, vol. 24, No. 1, pp51-53, March 2005.

[6] M. J. Long, D. F. Chen, J. Zhang and Q. Ouyang, "Novel online temperature control system with closed feedback loop for steel continuous casting," Ironmaking and Steelmaking, vol. 38, No. 8, 2011, doi:10.1179/1743281211Y.0000000042.

[7] B. W. Li, W. J. Chen and Y. Z. Zhang, "The Design and Application of Intelligent Temperature Control System Based on PID" Nanjing University of Science and Technology. Nanjing, vol. 2, pp. 5-7, September 2013.

[8] B. W. Li, W. J. Chen and Y. Z. Zhang, "Step-Shaped Temperature Control System Based on PID," Ordnance Industry Automation, vol. 30, No. 9, pp 83-85, September 2011, doi:10.3969/j.issn.10061576.2011.09.023.

[9] D. Bharadwaj and L.N. Das, "Design and Development and Hardware Implementation fo Fuzzy Knowledge Based Intelligent Temperature Control System," International Journal of Engineering Science and Technology, vol. 3, No. 6, June 2011, pp 4614-4625.

[10] N. Zhang, W. S. Xu, W. W. Wang, "Summary and Development and Application of Artificial Intelligent Technology," Coal Mine Machinery, vol. 30, No. 2, February 2009, pp 4-7, doi:10.13436/j.nkjx.2009.02.095.

[11] W. T. Ma, W. X. Yan, Z. Fu, Y. Z. Zhao, "A heating fuzzy control for Chinese cooking robots," Journal of Harbin Institute of Technology. Harbin, vol. 44, No. 1, January 2012, pp 120-125. 